\title{
2dF Spectroscopy of Globular Clusters in M104
}

\author{
Terry Bridges, Steve Zepf, Katherine Rhode, Ken Freeman \\ Anglo-Australian Observatory; Michigan State University; Yale \\ University; Australian National University
}

\begin{abstract}
We have found 56 new globular clusters in M104 from 2dF multifiber spectroscopy, doubling the number of confirmed clusters, and extending the spatial coverage to $50 \mathrm{kpc}$ radius. We find no significant rotation in the total sample, or for subsets split by color or radius. However, there are hints that the blue clusters have a higher rotation than the red clusters, and for counterrotation of clusters at large radius. We find a total mass of $\mathrm{M} \sim 1 \times 10^{12} \mathrm{M}_{\odot}$ and $\mathrm{a}(\mathrm{M} / \mathrm{L})_{B}=30$ out to $50 \mathrm{kpc}$ radius, which is strong evidence for a dark matter halo in M104.
\end{abstract}

\section{Data}

Globular cluster (GC) candidates were selected from KPNO mosaic BVR imaging (Rhode \& Zepf 2003, AJ, 126, 2307). We obtained a sample of 585 candidates with $19<\mathrm{V}<21.5$, after color cuts and image classification. In April 2002, we used the $2 \mathrm{dF}$ multi-fiber spectrograph on the AAT to obtain spectra for 200 candidates, which yielded 56 confirmed GCs.

\section{Globular Cluster Kinematics and Dark Matter in M104}

The velocity dispersion of the total sample is $\sim 200 \mathrm{~km} / \mathrm{s}$, with no significant difference between the blue and red GCs. A smoothed velocity dispersion profile shows that $\sigma$ decreases from $\sim 225 \mathrm{~km} / \mathrm{s}$ near the galaxy center to $\sim 125 \mathrm{~km} / \mathrm{s}$ at $50 \mathrm{kpc}$ radius.

The smoothed velocity profile shows rotation of $\sim 50 \mathrm{kpc}$; this is not statistically significant, however, and we place a 95\% upper limit on rotation of $110 \mathrm{~km} / \mathrm{s}$ for the total sample. We have looked for rotation amongst the blue and red GCs separately, and also for GCs at small and large radius (separated at $10^{\prime}=25 \mathrm{kpc}$ ). There is no significant rotation for any subsample. However, there are hints that the blue GCs have higher rotation than the red GCs, and that there is more rotation at large radius. Interestingly, the GCs at large radius may be counter-rotating with respect to the GCs and stars at smaller radius, but again this is not significant.

We have used the Projected Mass Estimator, assuming isotropic orbits, to find a total mass of $1.2 \times 10^{12} \mathrm{M}_{\odot}$ and a $(\mathrm{M} / \mathrm{L})_{B}=30$ at $50 \mathrm{kpc}$ radius. This is strong evidence for a dark matter halo in M104. 